\title{
The orienting response and direction of stimulus change
}

ALVIN S. BERNSTEIN, ${ }^{1}$ DEPARTMENT OF PSYCHIATRY, DOWNSTATE MEDICAL CENTER, STATE UNIVERSITY OF NEW YORK, Brooklyn, New York 11203

Following habituation to a given stimulus intensity, rearousal of the GSR OR was significantly greater to a stimulus change involving an increase in intensity than to an equal absolute amount of change involving a decrease in intensity. This was true both in normal and chronic schizophrenic samples. Thus, both the direction in which stimulus change occurs as well as the degree of change involved are important variables in triggering the $O R$.

The orienting response (OR) literature in general, and Sokolov's neuronal model theory in particular, stress the relationship between the $O R$ and the amount or degree of stimulus change encountered (Sokolov, 1960, 1963; Lynn, 1966). Sokolov postulates that mismatch between current stimulus input and internalized neuronal model is an essential agent in triggering any $\mathrm{OR}$, and that the greater the degree of mismatch, the greater the liklihood of an OR. For example, it has been shown that following habituation to a stimulus of given frequency or position (or intensity, or duration, etc.), the frequency of $O R$ rearousal increases the greater the difference between the rearousing and the habituated stimuli (Apelbaum et al, 1960; Sokolov, 1960).

However, in emphasizing the role of amount of change, the possibility that the direction of stimulus change may itself be a significant variable, independent of the amount of change involved, has so far been ignored. The present studies were conducted to determine whether direction of stimulus change exerted a differential effect in triggering an OR when the amount of change involved was equated across directions.

\section{EXPERIMENT 1 AND REPLICATION}

These investigations were part of a series of studies of the $O R$ in chronic schizophrenics (Bernstein, 1964, 1967, in press).

Subjects

Forty-eight healthy male controls (mean age 38.5, SD 8.24 years) and 120 male chronic schizophrenics (mean age 41.4, SD 9.32 years; mean length of hospitalization 11.7 years, SD 5.91 years) served as Ss.

Method

The Ss sat $3 \mathrm{ft}$ from a rear-projection screen in a semi-darkened, sound-deadened room. An assistant flashed a light in the instrument room whenever $S$ was not addressing the screen with eyes open; no stimuli were given while the light was on. The GSR was continuously recorded from the 1 st and 3rd fingers of the preferred hand using an ac-type instrument, the Airborne Instruments Tissue Resistance Monitor. After a 5-min adaptation period a tachistoscope began presenting $1-\mathrm{sec}$ eye-level flashes, approximately 6-3/8 in. square, at intervals between $15-60 \mathrm{sec}$. Half the Ss received a $5 \mathrm{ft}-\mathrm{c}$, half a $25-\mathrm{ft}-\mathrm{c}$ flash for 10 consecutive trials. On Trial 11 (T11), the rearousal trial, there was an unannounced switch in intensity. Thus, a $20 \mathrm{ft}-\mathrm{c}$ change occurred for all Ss, but half saw an increase (the $+20 \mathrm{ft}-\mathrm{c}$ group), and half a decrease (-20 ft-c group) in stimulus intensity, $O R$, scored as change in log conductance, was defined as a deflection of at least 500 ohms within 1-3 sec after stimulus onset. All records were independently scored by two judges, and the few instances of disagreement resolved by a third independent judge.

Since some Ss responded occasionally on the last habituation trials, rearousal was defined as a response on $\mathrm{T} 11$ whose $(\log C)$ amplitude was at least $150 \%$ that of any response on Trials 8,9 , or 10. Ss who gave a T11 OR of greater amplitude than any on Trials 8,9 or 10 , but not $50 \%$ greater, were eliminated.

Between 6-10 weeks after the initial study, Ss were rerun exactly as before. At retest, 140 Ss were available: 42 controls ( 21 each in + and $-20 \mathrm{ft}-\mathrm{c}$ groups), and 98 patients $(51$ in +20 , and 47 in $-20 \mathrm{ft}-\mathrm{c}$ groups).

Results and Discussion

In the first study three Ss in the -20 , and two in the $+20 \mathrm{ft}-\mathrm{c}$ groups were eliminated for the reason stated above. Of the remaining 82 Ss given an increase in stimulus intensity on T11, 30 $(36.6 \%)$ showed rearousal, while only $10(12.3 \%)$ did so of the 81 who received a decrease. This difference is highly significant: $\chi^{2}=$ $11.653, p<.001$. On retest, four $S s$ in the $+20 \mathrm{ft}-\mathrm{c}$ group were dropped. Of the remaining $64+20 \mathrm{ft}-\mathrm{c}$ Ss, $15(23.4 \%)$ showed OR rearousal on $\mathrm{T} 11$, while only two $(2.8 \%)$ of 72 did so following stimulus intensity decrease. Again, the difference is significant: $\chi^{2}=11.401, \mathrm{p}<.001$.

Patients and controls were combined in these analyses because there was no difference between them here. For example, considered by themselves, the chronic patients also displayed significantly more frequent rearousal of $O R$ following a +20 than following a $-20 \mathrm{ft}-\mathrm{c}$ change: $\chi^{2}=9.461 . \mathrm{p}<.01$ on initial test; $\chi^{2}=5.057, \mathrm{p}<.05$ on retest.

While these results illustrate the importance of direction of stimulus change for the OR, there is a problem in these data. Since stimuli of different intensities were administered to the + and -20 ft-c groups on the crucial rearousal trial these data may confound the effect of direction of change with that of absolute intensity itself (e.g., Uno \& Grings, 1965). Another study was designed to eliminate this source of confusion.

\section{EXPERIMENT 2}

\section{Subjects}

Twelve healthy males (mean age 46.1 , SD 12.2 years) and 36 male chronic schizophrenics (mean age 41.7, SD 9.0 years; mean hospitalization 13.5 years, SD 6.7 years) were used as Ss.

Method

Procedure and scoring were the same as in the previous studies except that a dc Fels Dermohmmeter was used, and Ss wore headphones. After the adaptation period, half the sample received a $1000 \mathrm{cps}$ tone binaurally for $1 \mathrm{sec}$ at $60 \mathrm{~dB}$, the other half at 90 $\mathrm{dB}$ (re .0002 dynes $/ \mathrm{cm}^{2}$ ). This was repeated 15 times, irregularly as before. On Trial 16 (T16), the rearousal trial, all samples received the same 1000 cycle tone at $75 \mathrm{~dB}$. There was thus a change of either +15 or $-15 \mathrm{~dB}$ to a single, common rearousal signal.

Results and Discussion

One $S$ in the $-15 \mathrm{~dB}$ group was eliminated. Here again, chronic schizophrenics and controls were similar with regard to the influence of direction of change on the $O R$ and their data were combined. Of the $24 \mathrm{Ss}$ responding to $75 \mathrm{~dB}$ after exposure to 60 $\mathrm{dB}$ (the $+15 \mathrm{~dB}$ group). 14 showed an OR on T16 (58.3\%); of the 23 Ss responding after exposure to $90 \mathrm{~dB}(-15 \mathrm{~dB}$ group), only three showed rearousal (13.0\%). Again, the difference is highly significant: $\chi^{2}=8.564, p<.005$, and again, the results were the same when the patients were examined alone: $\chi^{2}=7.127, p<.01$.

In both normal and patient samples response to $90 \mathrm{~dB}$ was of greater amplitude and took longer to habituate than did that to 60 dB. It was thus possible that the +15 and $-15 \mathrm{~dB}$ groups were at different levels of arousal at the time the crucial $75 \mathrm{~dB}$ signal was given, and that this might account for the difference in rearousal frequency.

This was studied by examining base resistance levels (BRL) in the + and $-15 \mathrm{~dB}$ samples. Three of the $48 \mathrm{Ss}$ were Negroes (all $+15 \mathrm{~dB})$. Since BRL among Negroes is higher than among whites (Johnson \& Corah, 1963; Bernstein, 1965), these three were not included. BRL was averaged to the nearest $500 \mathrm{ohms}$ for each of the remaining Ss over the $10 \mathrm{sec}$ just preceding T16. Median BRL for the $+15 \mathrm{~dB}$ group was $113.0 \mathrm{~K}$ ohms (range 19.5-355.5 K ohms), while the median in the $-15 \mathrm{~dB}$ group was $112.0 \mathrm{k}$ ohms (range 36.5-290.5 K ohms). By Mann-Whitney test these two groups do not differ significantly $(z=0.469, \mathrm{p}>.60)$, and the difference in $O R$ rearousal frequency cannot readily be attributed to differences in prestimulus-change arousal level.

Thus, with the earlier source of confusion eliminated, these data permit a more definite conclusion: a stimulus change from weaker to stronger intensity triggers a significantly greater frequency of (GSR) OR than does an equal absolute amount of change in the opposite direction. Similar GSR data have been reported by Grings (1960) working with the "perceptual disparity response." How- 
ever, in terms of orienting response neuronal model theory, Russian authors have primarily stressed a "quantity-of-change" bias in the model's function as a selective filter; i.e., more change yields more $O R$ and a more reliable facilitation of perception (Sokolov, 1960). The present data suggest that a "direction-ofchange" bias is also present, in chronic schizophrenics as well as the general population. It remains to be seen, however, whether this increased OR response to "greater-than" changes is specific to the intensity dimension or more generally true of other stimulus parameters (and other physiological measures) as well. For example, it is possible that such directional OR bias may represent a heightened sensitivity to the class of stimuli indicating "something approaching the organism." Such sensitivity to things approaching one's self seems functionally advantageous; it also generates testable hypotheses, and work has begun to carry out such tests.

\section{REFERENCES}

APELBAUM, J., SILVA, E., FRICK, O., \& SEGUNDO, J. Specificity and biasing of arousal reaction habituation. EEG clin. Neurophysiol., 1960, 12, 829-840.

BERNSTEIN, A. S. The galvanic skin response orienting reflex among chronic schizophrenics. Psychon. Sci., 1964, 1, 391-392.

BERNSTEIN, A. S. Race and examiner as significant influences on basal skin impedance. J. Pers. Soc. Psychol., 1965, 1, 346-349.

BERNSTEIN, A. S. Electrodermal base level, tonic arousal, and adaptation in chronic schizophrenics. J. abnorm. Psychol., 1967, 72, 221-232.

BERNSTEIN, A. S. The orienting reflex as a research tool in the study of psychotic populations. In I. Ruttkay-Nedecky, L. Ciganek, V. Zikmund, and $\mathrm{E}$. Kellerova (Eds.), Mechanisms of the orienting reaction in man. Slovak Academy of Science, in press.

GRINGS, W. W. Preparatory set variables related to classical conditioning of autonomic responses. Psychol. Rev., 1960, 67, 243-252.

JOHNSON, L. C., \& CORAH, N. Racial differences in skin resistance. Science, 1963, 139, 766-767.

LYNN, R. Attention, arousal and the orientation reaction. New York: Pergamon Press, 1966.

SOKOLOV, E. N. Perception and the conditioned reflex. New York: MacMillan, 1963.

UNO, T., \& GRINGS, W. Autonomic components of orienting behavior. Psychophysiology, 1965, 1, 311-321.

\section{NOTES}

1. This paper was written during the tenure of NIMH Special Research Fellowship No. 1-F3-MH-34,080, and was presented in part to the Eastern Psychological Association Convention, April, 1967. The author is indebted to Kenneth Taylor for his invaluable aid in collecting and scoring data. 